\title{
Prevalence of coronavirus disease 2019 (COVID-19) among healthcare professionals working in hospital emergencies during the first-wave peak in 2020 in Porto Alegre, Brazil
}

\author{
Ana Luisa F. Dubiela BSc ${ }^{1}$, Daiane F. Dalla Lana PhD , Ana Paula K. Aerts MD², Cristiani G. de Marques BSc ${ }^{2}$, \\ Renato Cassol MD³ , Micheline G. Dalarosa RN³ , Vanessa Schultz MD ${ }^{4}$, Fabiano Ramos MD ${ }^{5}$, Isabelli Guasso RN ${ }^{5}$, \\ Fabio F. Dantas Filho MD, PhD ${ }^{6}$, Silvana T. Dal Ponte $\mathrm{MD}^{6}$, João Carlos B. Santana MD, PhD ${ }^{6}$, \\ Michelle D. Santarem RN, PhD ${ }^{6}$, Morgana P. de Camargo RN ${ }^{6}$, Maria Luiza P. Machado RN, PhD 6 , \\ Teresa Cristina T. Sukiennik $\mathrm{MD}^{2}$, Guilherme Watte $\mathrm{PhD}^{1}$, Antonio N. Kalil MD, $\mathrm{PhD}^{1,2}$ and \\ Alessandro C. Pasqualotto MD, $\mathrm{PhD}^{1,2}$ (1) \\ ${ }^{1}$ Universidade Federal de Ciencias da Saude de Porto Alegre, Porto Alegre, Brazil, ${ }^{2}$ Santa Casa de Misericordia de Porto Alegre, Porto Alegre, Brazil, ${ }^{3}$ Hospital \\ Nossa Senhora da Conceição, Porto Alegre, Brazil, ${ }^{4}$ Hospital Mae de Deus, Porto Alegre, Brazil, ${ }^{5}$ Hospital Sao Lucas of Pontifical Catholic University of Porto \\ Alegre, Porto Alegre, Brazil and ${ }^{6} \mathrm{Hospital}$ de Clinicas de Porto Alegre, Porto Alegre, Brazil
}

To the Editor - Brazil ranks third among countries with the highest number of coronavirus disease 2019 (COVID-19) cases in the world, with 11.5 million documented infections as of March 2021. Healthcare professionals (HCPs) are possibly the occupational category at the highest risk for severe acute respiratory coronavirus virus 2 (SARS-CoV-2) exposure. We determined the seroprevalence of SARS-CoV-2 among HCPs working in hospital emergencies in Southern Brazil during the first-wave peak in 2020.

\section{Methods}

In this cross-sectional study, we evaluated the prevalence of COVID-19 in HCPs working in the emergency departments of 5 large tertiary-care hospitals located in Porto Alegre, Southern Brazil (population 1.5 million). HCPs were evaluated on July 20-24, 2020, and again after 3 weeks, in August 10-14, 2020. At each encounter, clinical data were obtained and a blood sample was taken by finger pricking for antibody detection (Standard Q COVID-19 IgM/IgG Duo-Biosensor, South Korea). Data were analyzed using Statistical Package for the Social Sciences version 22.0 software (IBM, Armonk, NY). Categorical variables were expressed as absolute and relative frequencies. Continuous variables were presented as mean values \pm standard deviations (SD).

\section{Results}

In the first phase of the study, 1,163 HCPs were evaluated $(87.1 \%$ of study population). Most were woman (66.6\%), and the median age was 38 years (SD, \pm 10 years). Professional roles included nursing assistants (43.5\%), physicians (23.0\%), nurses (15.0\%), administrative workers $(12.9 \%)$, and cleaners $(3.6 \%)$. The most frequent chronic health conditions among these individuals were asthma $(8.0 \%)$, arterial hypertension $(7.9 \%)$, rhinitis $(2.4 \%)$, hypothyroidism $(2.3 \%)$, and diabetes mellitus (1.5\%).

Author for correspondence: Alessandro C. Pasqualotto, E-mail: pasqualotto@ santacasa.org.br

Cite this article: Dubiela ALF, et al. (2022). Prevalence of coronavirus disease 2019 (COVID-19) among healthcare professionals working in hospital emergencies during the first-wave peak in 2020 in Porto Alegre, Brazil. Infection Control \& Hospital Epidemiology, 43: 956-957, https://doi.org/10.1017/ice.2021.139
Nearly all study participants reported the use of individual protection equipment, including masks (99.8\%), face shields (90.3\%), and gloves (85.6\%). Known exposure to COVID-19 patients was reported by $82.3 \%$ of the HCPs, mostly in June 2020 (62.3\%). Most HCPs had been asymptomatic during the COVID-19 pandemic (58.2\%), while some reported fever (11.7\%), shortness of breath $(27.8 \%)$, and cough (11.6\%). A few of these HCPs had laboratory-confirmed COVID-19 in June (2.8\%) and July (2.2\%).

In the first phase of the study, $5.5 \%(n=64)$ were found to have antibodies against COVID-19: 26 had IgM type, 19 had IgG type, and 19 had both. Of these 78 HCPs, 27(34.6\%) had been previously diagnosed with COVID-19. Marked variation was observed among hospitals, regarding COVID-19 seroprevalence (Fig. 1). After 3 weeks, 911 individuals (78.3\% of original sampling) returned for testing (study phase 2 ), and $5.6 \%$ tested positive for an antibody: 17 for $\operatorname{IgM}, 17$ for IgG, and 17 for both. $\operatorname{IgM}$ became negative in the second study evaluation in $55.3 \%$ of participants who had previously tested positive for these antibodies, and IgG became negative in $50.0 \%$ who had previously tested positive.

\section{Discussion}

This is the first study to evaluate the prevalence of SARS-CoV-2 in HCPs in Brazil. Previous studies conducted elsewhere have addressed the question, mostly using real-time polymerase chain reaction (PCR) tests. The occupational health service of Massachusetts performed a study to assess COVID-19 prevalence in HCPs, revealing that $14.0 \%$ had a positive PCR test at the initial evaluation. ${ }^{1}$ In Hong Kong, $29 \%$ of HCPs were found to be infected using PCR. ${ }^{2}$ In 2 Dutch hospitals, $6 \%$ of HCPs were infected with SARS-CoV-2 in March 2020. ${ }^{3}$ However, conducting epidemiological surveys with PCR is not practical because PCR results reflect viral detection at the moment of sampling only. Alternatively, SARS-CoV-2 prevalence can be determined by antibody detection. In Italy, a study showed that $14.4 \%$ of HCPs working in the hospital had detectable IgM antibodies against SARS-CoV-2. In the New York city area, a study conducted in June 2020 showed a $13.7 \%$ prevalence of SARS-CoV-2 antibodies in HCPs. ${ }^{5}$ In a 

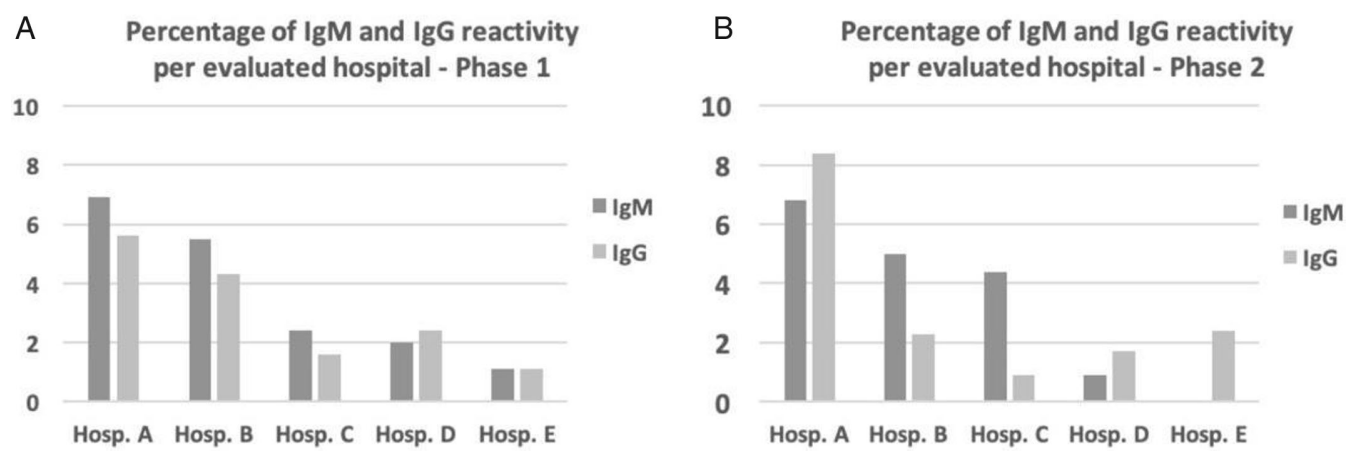

Fig. 1. Positivity for COVID-19 IgM and IgG antibodies in the first (A) and second (B) phases of the study, in the 5 hospitals studied. Hospitals are not identified in this slide, they are randomly named A-E.
Financial support. This study was sponsored by Instituto Cultural Floresta. We are in debt with Claudio Goldsztein and Marcelo Wallauer for supporting this study, in addition to all donors to Instituto Cultural Floresta.

Conflicts of interest. All the authors declare no conflicts of interest related to this article.

\section{References}

1. Lan FY, Lan FY, Filler R, et al. COVID-19 symptoms predictive of healthcare workers SARS-CoV-2 PCR results. PLoS One 2020;15(6):e0235460.

2. Wong SCY, Kwong RT, Wu TC, et al. Risk of nosocomial transmission of coronavirus disease 2019: an experience in a general ward setting in Hong Kong. J Hosp Infect 2020;105:119-127.

3. Bergh MFQK- Van Den. SARS-CoV-2 infection in 86 healthcare workers in two Dutch hospitals in March. medRxiv 2020. doi: 10.1101/2020.03.23. 20041913.

4. Sotgiu G, Barassi A, Miozzo M, et al. SARS-CoV-2 specific serological pattern in healthcare workers of an Italian COVID-19 forefront hospital. BMC Pulm Med 2020;20:203.

5. Moscola J, Sembajwe G, Jarrett M, et al. Prevalence of SARS-CoV-2 antibodies in healthcare personnel in the New York City area. JAMA 2020;324: 893-895.

6. Malfertheiner S, Brandstetter S, Roth S, et al. Immune response to SARS$\mathrm{CoV}-2$ in healthcare workers following a COVID- 19 outbreak: a prospective longitudinal study. J Clin Virol 2020;130:104575.

7. Long QX, Tang XJ, Shi QL, et al. Clinical and immunological assessment of asymptomatic SARS-CoV-2 infections. Nat Med 2020;26:1200-1204.

8. Liu A, Wang W, Zhao X, et al. Disappearance of antibodies to SARSCoV-2 in a COVID-19 patient after recovery. Clin Microbiol Infect 2020; 26:1703-1705.

9. Paiva KJ, Grisson RD, Chan PA, et al. Validation and performance comparison of three SARS-CoV-2 antibody assays. J Med Virol 2021;93:916-923.

\title{
Vaccines in the coronavirus disease 2019 (COVID-19) era: Game theory applications
}

\author{
Marios Papadakis MD, MBA, $\mathrm{PhD}^{1}$ (i) and Nikolaos Spernovasilis MD, MPH \\ ${ }^{1}$ University Witten-Herdecke, Germany and ${ }^{2}$ School of Medicine, University of Crete, Heraklion, Greece
}

To the Editor-Vaccines are among the greatest inventions of science, preventing millions of deaths worldwide annually. Currently, the lack of a coronavirus disease 2019 (COVID-19) vaccine has led to

Author for correspondence: Marios Papadakis, E-mail: marios_papadakis@yahoo.gr Cite this article: Papadakis M and Spernovasilis N. (2022). Vaccines in the coronavirus disease 2019 (COVID-19) era: Game theory applications. Infection Control \& Hospital Epidemiology, 43: 957-958, https://doi.org/10.1017/ice.2021.125 a pandemic that has brought the whole world to a near standstill. Although international collaborations to guarantee equitable access have been established, as COVID-19 vaccines are approved, national egotism is expected. ${ }^{1}$ Game theory is the science of strategy and interactive decision making, where the outcome depends not only on one's actions but also on the actions of others. ${ }^{2}$ Herein, we present applications of game theory in vaccine allocation. 